\title{
Systematic Studies of the LEP working point
}

\author{
P. Collier and H. Schmickler, \\ CERN, Geneva, Switzerland
}

\begin{abstract}
The working point, i. e. the fractional part of the betatron tunes is one of the key parameters for machine operation. This paper reports on systematic studies in LEP on the dependence of beam sizes and beam lifetimes on betatron tunes. For these studies the horizontal and vertical tunes have been varied by an automatic measurement sequencer over the wide range 0.1 to 0.4 and beam sizes and lifetimes have been recorded in parallel.
\end{abstract}

\section{Measurement Procedure}

During a tune scan the betatron tunes of LEP are changed by triming the main quadrupole strings. The available trim range is $\Delta \mathrm{q}_{\mathrm{h}}=\Delta \mathrm{q}_{\mathrm{v}}= \pm 0.15$ with a resolution of 0.001 . The maximum number of trim steps is 400 and the time interval between tune changes is 2 seconds., hence a complete scan takes about 12 minutes measurement time. Synchronous to the tune variations several beam parameters are recorded. The tunes themselves are tracked by the LEP q-meter in PLL mode [1]. The transverse beam dimensions are recorded from the UV-monitors and beam lifetimes from the bunch current transformers.

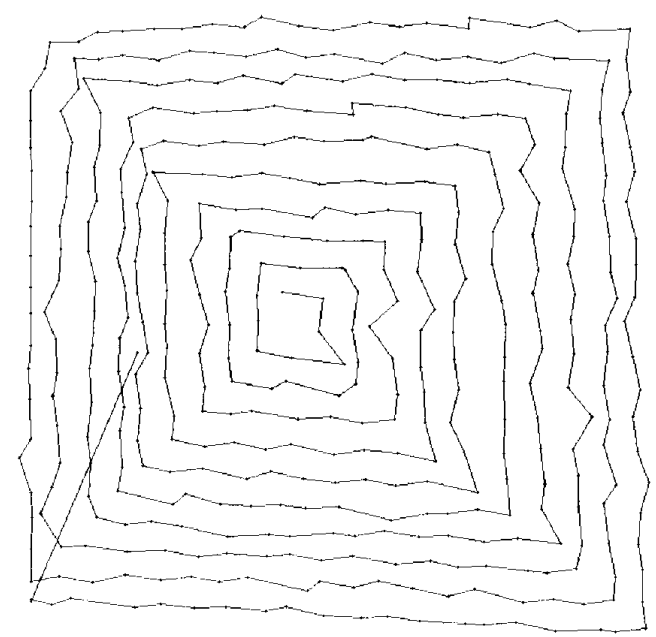

Figure. 1. Trace of measured tunes during a tune scan; horizontal axis: measured $\mathrm{q}_{\mathrm{h}}$; vertical axis: measured $\mathrm{q}_{\mathrm{v}}$

Fig. 1 shows the measured tunes during a tune scan. Consecutive points of the scan are connected with a line. Optionally the scan pattern could be rotated by $45^{\circ}$ in order to cross specific beam resonances faster.

All data sets are retrieved and merged for further processing. More details on the measurement procedure can be found in [2] and references therein.

\section{Available data sets}

During the LEP running period 1994 systematic studies of the working diagram were performed. The following machine conditions were of interest: The untuned lattice at injection energy (beam energy 20 $\mathrm{GeV}, \beta_{\mathrm{v}}^{\star}=21 \mathrm{~cm}$ ) and the tuned lattice at collision energy (beam energy $45.6 \mathrm{GeV}, \beta_{\mathrm{v}}^{\star}=5 \mathrm{~cm}$ ). As LEP was operated in 1994 with horizontal pretzel orbits comparative measurements were done by performing tune scans with the pretzel orbits on or off. None of the available data sets contains beams in collision. Even at moderate bunch currents colliding beams suffer from many more strong resonances and no consistent data set could be recorded over a wide tune range for colliding beams. As an example, fig. 1 on the last page of this paper shows the inverse of vertical and horizontal beam sizes as a function of the measured tunes. In the top picture one can clearly see the main coupling resonance $\mathrm{q}_{\mathrm{h}}=\mathrm{q}_{\mathrm{v}}$ and its synchrotron sidebands $\left(q_{s}=0.065\right)$. Furthermore the synchro-betatron resonance $\mathrm{q}_{\mathrm{v}}=2 \cdot \mathrm{q}_{\mathrm{s}}$ is strongly seen and we see an indication of the third order resonances $\mathrm{q}_{\mathrm{v}}=2 \cdot \mathrm{q}_{\mathrm{h}}$ and $\mathrm{q}_{\mathrm{h}}=2 \cdot \mathrm{q}_{\mathrm{v}}$ respectively.

\section{Search for a new working point}

The principle goal of the above studies was the search for a working point for physics giving good lifetime conditions and high luminosities. As stated above tune scans do not give valuable data with beams in collision. Hence the search for a working point has to happen in several steps. First the largest possible aera in tune space is measured with single beams and favourable regions in terms of lifetimes and small beam sizes are identified. Thereafter the following considerations are added:

- the working point point for injection energy and collision have to be close in order to avoid the necessity of jumping strong resonances during energy ramping or beta squeezing

- results of compute simulations of the beam-beam effect are considered [4] and [5]

- the working point should be far from the coupling resonance $[3]$. 


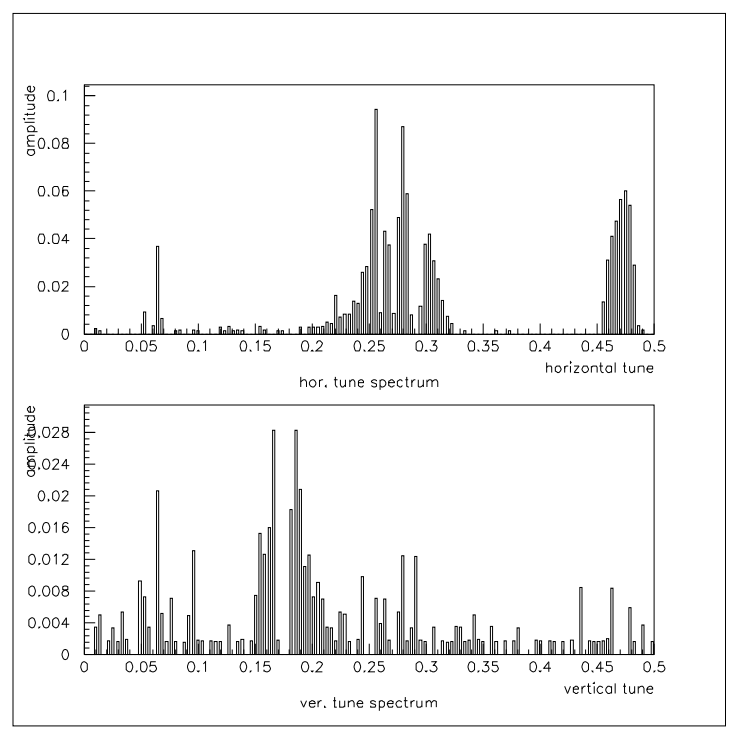

Figure. 2. Measured tune spectra with LEP beams in collision

- beams in collisions do not have unique betatron tunes, but due to the nonlinear nature of the beam-beam force the beams cover a whole aera in tune space. The whole aera covered should be free of resonances.

The above effect of the beams in collision covering a certain aera in tune space (a so called footprint) has been studied with computer simulations for LEP [6]. Additional information can be obtained from experimental observations. Fig. 2 shows a typical spectrum of the transverse tunes of LEP beams in collisions. Several coherent beam-beam modes are visible apart from the undisturbed tunes $\mathrm{q}_{\mathrm{h}}=0.28$ and $\mathrm{q}_{\mathrm{v}}=0.16$. Taking this information, a random number generator can be constructed that generates a particle population in tune space according to the distributions measured in fig. 2. The resulting distribution, called a coherent footprint is shown in fig. 3. An overlay of this information to the the measured tune scans gives additional information for the selection of a working point.

Taking together all arguments the following three working points have been tested experimentally:

$$
\begin{aligned}
& \text { 1: } \mathrm{q}_{\mathrm{h}}=0.28 \text { and } \mathrm{q}_{\mathrm{v}}=0.16 \\
& \text { 2: } \mathrm{q}_{\mathrm{h}}=0.23 \text { and } \mathrm{q}_{\mathrm{v}}=0.36 \\
& \text { 3: } \mathrm{q}_{\mathrm{h}}=0.36 \text { and } \mathrm{q}_{\mathrm{v}}=0.16
\end{aligned}
$$

Working point 2 and 3 had lower beam sizes with separated beams, but as soon as high bunch currents were collided the beam lifetimes became bad and large particle backgrounds were seen by the LEP experiments. Finally the "standard" working point 1 choosen already for the running period 1992 is still used in daily operation.

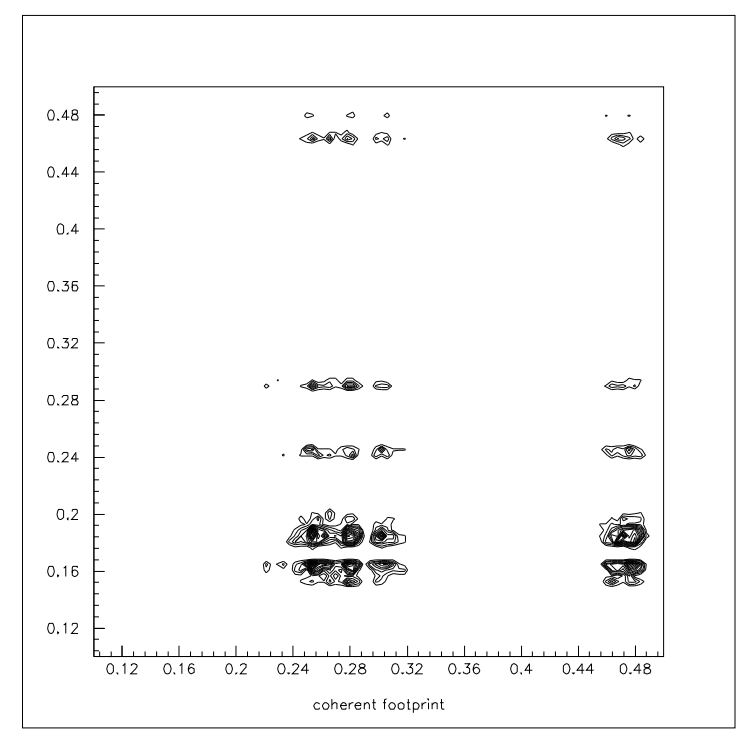

Figure. 3. Computer generated distribution of tune space occupied by particles in collision. A random number generator has been constructed to generate a footprint of the particle according to the measured coherent spectra from above

\section{Conclusions}

A measurement sequencer that records beam parameters as a function of the betatron tunes is a very valuable for beam diagnostics and the understanding of the machine lattice. It is very difficult to interpret the data for luminosity optimization. Here the measured data provide starting information that has to be complemented with experience and intuition.

\section{References}

[1] K. Lohmann, M. Placidi and H. Schmickler Q-Monitoring in LEP, LEP-BI Note 88-45.

[2] H.Schmickler,"Tune Scans", Proc. of the Third Workshop on LEP Performance, Chamonix 1993.

[3] P. Collier and H. Schmickler, "The Influence of the Vertical Closed Orbit on Luminosity Performance in LEP", Proc. EPAC, London, 1994.

[4] E. Keil, Nucl. Instr. Meth. 188 (1981) 9. E. Keil, LEP Note 636 Rev. (1991). E. Keil, LEP Performance Note 86 .

[5] S. Myers, "Simulation of the Beam-beam Effect in Storage Rings", Nucl. Instr. Meth. 211(1983) 263.

Y. Marti, S. Myers, J. Poole, SL Note 92-50 (DI)

[6] Werner Herr, "Can we learn anything from beam-beam footprints?"

Proc. of the Fourth Workshop on LEP Performance, Chamonix 1994. 